\title{
IOT BASED PARALYSIS PATIENT HEALTH CARE MONITORING SYSTEM
}

\author{
${ }^{1}$ Ms.N.ReneeSegridReddiyar, ${ }^{2}$ S.Remina, ${ }^{3}$ S.Sabrin, ${ }^{4}$ M.Subhashini \\ ${ }^{1}$ AssistantProfessor, ${ }^{2,3,4}$ U.GScholar,DepartmentofElectronics and communication Engineering, \\ FrancisXavierEngineeringCollege,Tirunelveli
}

Email : reneereddiar@gmail.com, reminas523@gmail.com, sabrin072000@gmail.com,subhashini10599@gmail.com

\begin{abstract}
We all know that the paralysis condition is a loss of muscle function in the body parts. It can affect any part of your body at any time, then probably you may won't feel the pain the affected area. Technical and Therapautic innovations are there to improve the quality of life Our goal is to develop a device which should be easy to use and should be affordable which consists of basic health care monitoring system with nursing care. We know that these people can't able to convey their messages or needs. To overcome this, we come up with the system that helps these patients to display messages by very simple motion. This device can be designed to be mounted on the finger or to be inbuilt in their clothes.
\end{abstract}

KEYWORDS:datapre-

processing, mapping technique,classification using KNN algorithm, Arduino UNO micro controller, hand gestures, SVM classifier,accelerometer, dynamic time warping (DTW).

\section{Introduction}

The paralysis patients are unable to move their muscles for their purposes. There are so many symptoms and causes for this condition, especially spinal cord injury which affects the nervous system. There are some existing systems for individual comforts. But this system will help to monitor the overall need of the patients. Their messages will be displayed on the LCD screen. In this, we are also having some sensors. The aim is to purpose a novel device which helps disabled people. It will help them to interact with other people with minimum efforts. This device may one day improve the lives of the people with paralysis.Even though, there are so many innovative approaches for curing these people, but here this will help them to adapt with paralysis by making them as independent as possible. Fortunately, the last decade has seen promising technology advances to address these concerns. In addition, the accelerometer will also give a buzzer sound when patients fall on the floor.

\section{HardwareImplementation}

There are several existing systems available for patients with paralysis but this system helps to constantly monitor and understand the patient's needs. The sensor in the system aids to transmit the patient's message,and the message is displayed onthe LCD display. The message will 
change according to the position of the accelerometer. We must then know their needs and assist them on the basis of their needs. The temperature sensor, humidity sensor and pulse meter were used in this system. These sensors should be for patients witht issues, orgloves; they can feel the temperature of the patient, moisture and pulses.If the patient is in a critical situation, it will sound alert with a buzzer when the patient is on thefloor or when the pulse speed is above normal levels.This system can help treating patients suffering from paralysis, and it's also very cheap and easy to buy without debts.

\section{ProposedSystem:}

\subsection{Block Diagram:}

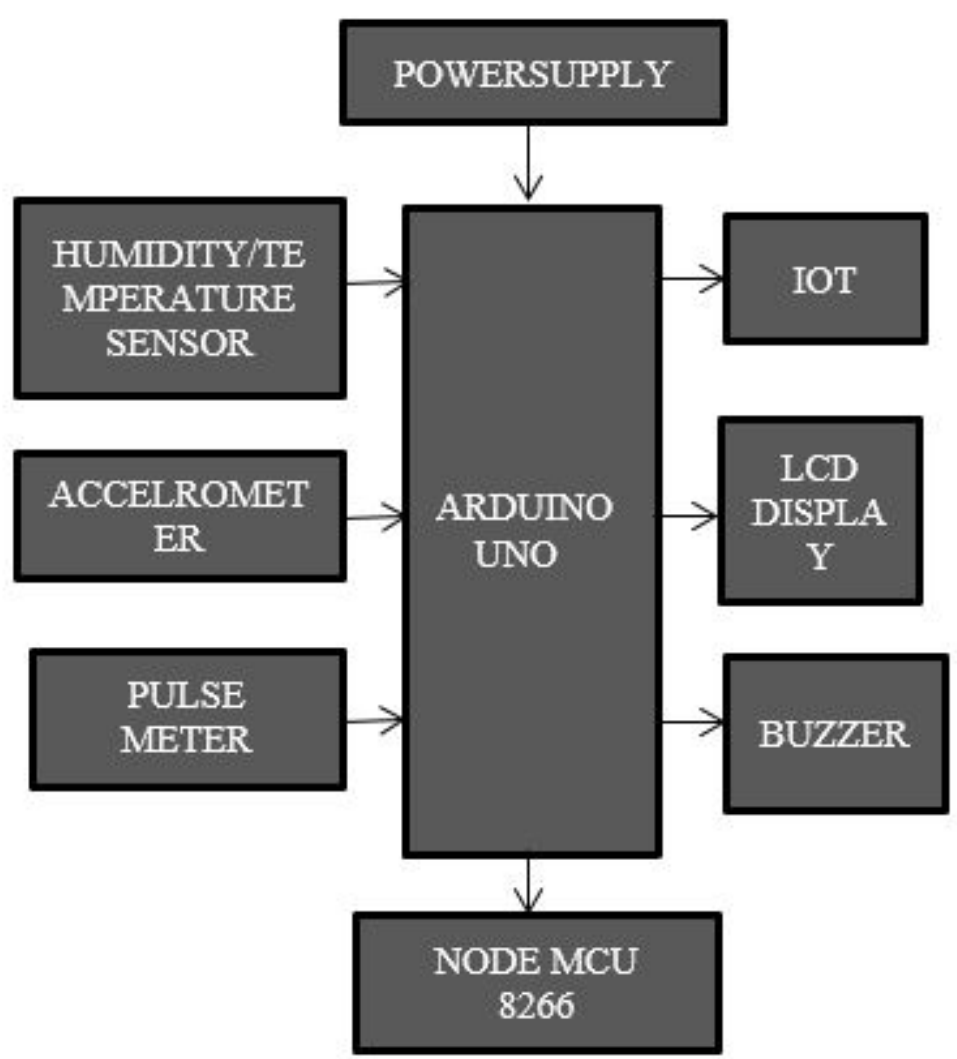

\subsection{Procedure}

Figure 1. Block Diagram

The IOT just is the web of stuff. Independent technologies can collect and transmit data without human intervention via a wireless network.There are endless personal or business opportunities. In the health care industry, remote monitoring has been possible with IOT-enabled devices that releasethe possibility of keeping patients safe and healthy and that enable doctors to provide superlative health care. Respond to and enable physical objects to collect information and respond to instructions. To collect, store, handle, manipulate, and manipulate data. 
The communications infrastructure that includes protocols and technologies that permit two physical objects to exchanged ataismostimportant. Adafruit IO is a simple and easy-to-use IOT platform for data storage,data viewing and device control. By using the adafruit web page, this project output is displayed on the mobile. Connect the respective sensors to a node MCU which gets connected to a webpage i.e. adafruit io. By using thiswebpage, we can monitor the data gathered by the sensors. Steps to create adafruit account. Click the sign in button in the top right corner of adafruit.com After that, type the emailid, username and password.Adafruit account will be successfully created. Create the widgets as your wish.Connect the respective sensor which gets connected to abuzzer. Based on the patient needs it will give soundand the output will be displayed on the LCD display and if the patients pulse or temperature become abnormal it will be displayed on the webpage which will be monitored continuously.

\section{Result}

\subsection{CircuitConnection}

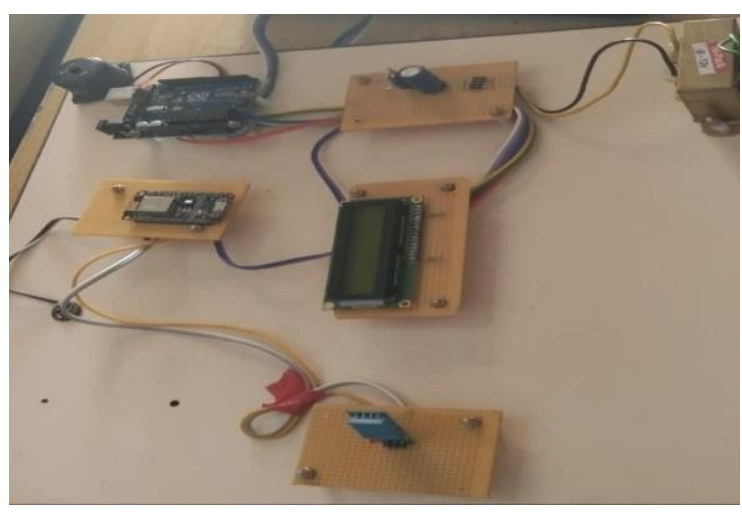

Figure 2Circuit Connection

This is our final circuit connection.There lated message has now been shown on the LCD panel by the help of micro controller 


\subsection{LCDDisplay-Nothing}

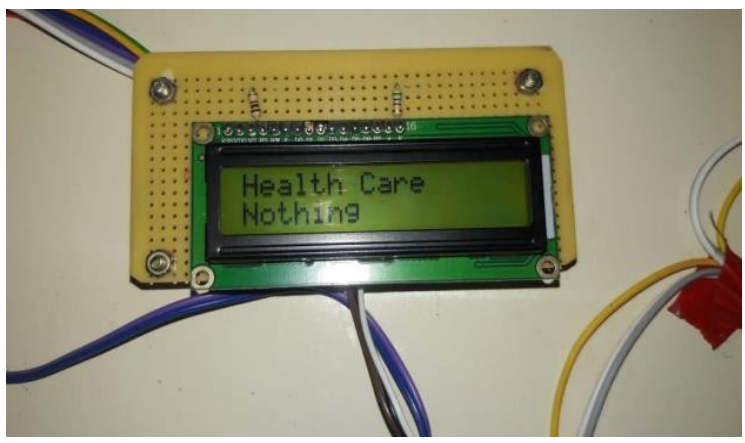

Figure 3 Lcd Display-Nothing

The microcontroller has now displayed there later message on the LCD panel. As a consequence, if there is no movement in the patient. Nothing will be shown.

\subsection{LCD Display-Need Food}

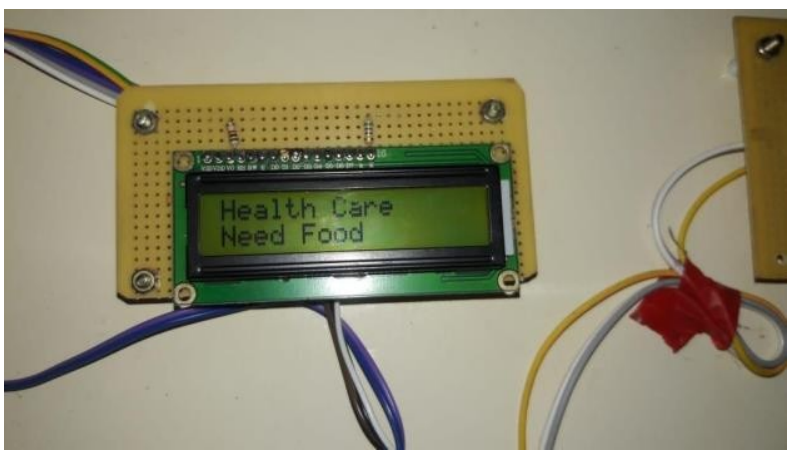

Figure 4LcdDisplay-NeedFood

The performance will be shown depending on the patient's needs. If the accelerometer is set to acertain angle, it will mean that they need food and sound an alarm.

\subsection{LCDDisplay-NeedWater}

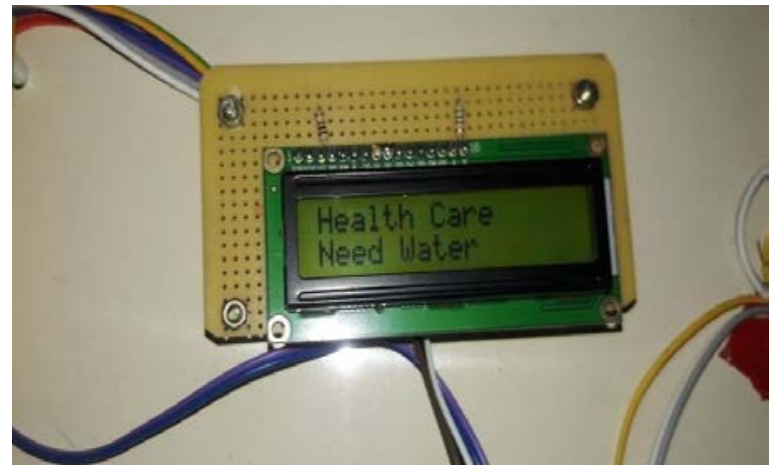

Figure5LcdDisplay-NeedWater

The displayed outputs will assist patients in meeting their needs. They need water or their requir ements if the angle changed based on their need it will alert the persons. 


\subsection{LCD Display-GoingtoToilet}

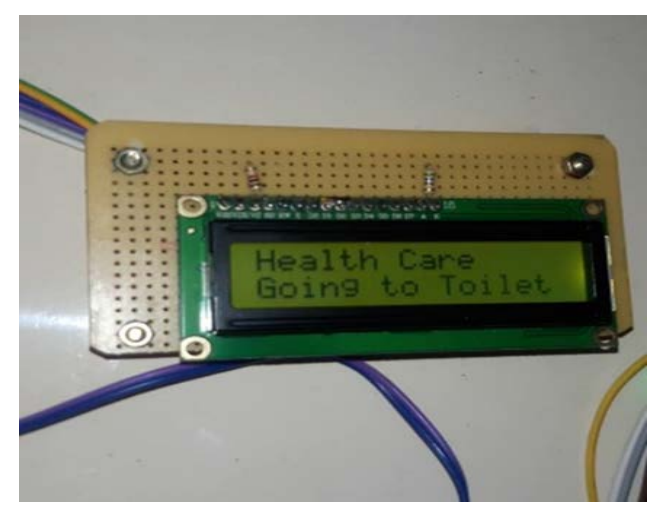

Figure6LcdDisplay-GoingtoToilet

The patient's health will be continuously monitored. If they need to go restroom then they can tilt their hand in a particular position, it will be shown on the LCD display

\subsection{LCDDisplay-Emergency}

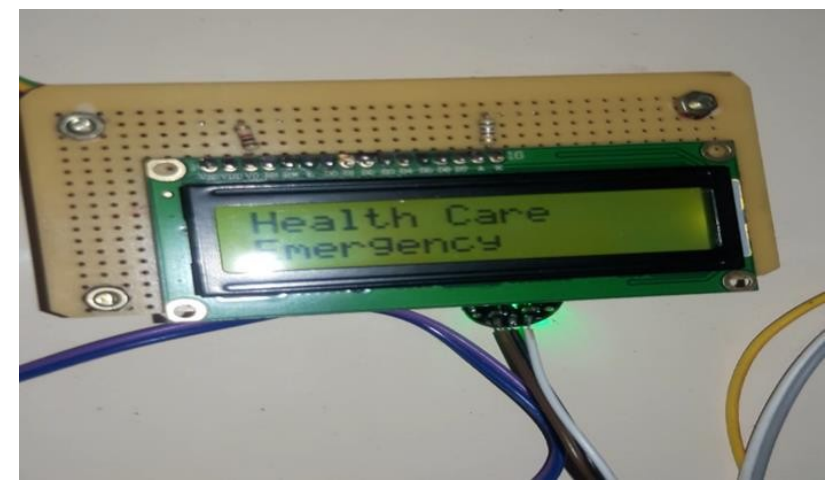

Figure3.6LCDDisplay-Emergency

Pulse rate and body temperature will be registered, as well as fear,so that if the patient is in an emergency,or else if they fall down on the floor the in formation will be shown automatically and a continuous warning sound will be produced through buzzer.

\subsection{Adafruit}

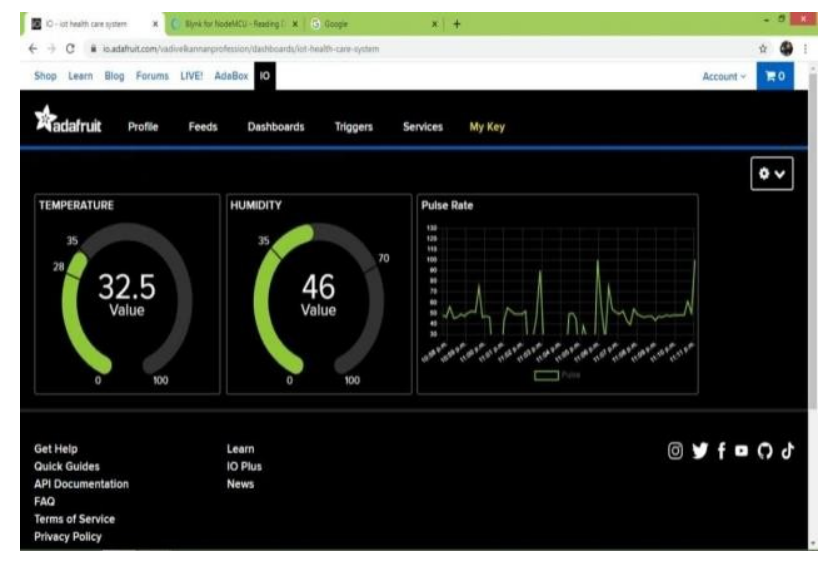

Figure 3.7AdafruitOutput 
Adafruit IOT is an easy-to-use IOT platform that is useful for storing data,viewing data,and controlling devices. We can use adafruit to trackand manage stuff. It will constantly monitor the patient's health and save their records. In this project pulse rate and temperature and humidity will show on mobile screen using adafruit webpage.

\section{Conclusion}

This system is really helpful for paralyses patients. When they need help then they can ask by using some movements they can also survive in this world like normal people by using this movement detection. This system is reliable and cheap and less weight.so they can buy without debt.This system will make paralyses patients to achieve a independent of mobility. This is not a trivialtask just because it varies from person to person in its nature and type.Therefore,different methods are essential to support these people, and it is our duty,as future engineers,to develop new technologies to help paralysed patients.

\section{FutureScope}

In future, we can use the chipset to implement thissystem. All parts are integrated in the chip, so thatwe can. This chip fits easily with the patient withparalysis Glovesand bandsavoid clothes. But thereis one disadvantage that will happen increase costbut theincrease.

\section{REFERENCES}

[1] PatientMotionUsing[1]RolandAuberger,MichaelFriedrichRussold,RobertRiener, HansDietl,2019"Everydaycomputerisedlegbrace"IEEETransactionsonLocomotiv eTasks“BionicsandMedicalRobotics,vol.1,no.2,pages 106-114.

[2] Varun G. Menon, Fadi Al Turjman, Vinoj P. G.,LeonardoMostarda,2019"DeepLearningIntelligenceSystemsAssistanceandPost -Stroke"IEEEAccess,Vol7,pp.Rehabilitation."Thailand-133463.

[3] TingWang,ShuZhang,FacialAutomaticParalysisLiu,GengkunWu,JunyuDong,201 9"AssessmentEnhancedbyaCascadeStructureNetwork Encoder" V.7,pp. 135621 135631 Access, vol.

[4] Xiaohua Tian, Xuesheng, Yisheng Ji, Tianyi Wang,SijieXiong, Xinbing, Binyao Jiang, Sijie Xiong. AWearable Device Wang,2019"iBlink: Facial 
ParalysisEnablingPatientsintheBlink',IEEEMobileTransactionsVol.17891801,No.8,pp.

[5] Zhexiao Guo , Georges Dan, Jianghuai Xiang, JunWang, Wanzhang Yang, Oliver Deussen, Huijun Ding,Yongjin. Zhou,2018"To the Disappointed FrameworkforcomputerisedevaluationUnilateralfacialperiphery "IEEE Biomedical and Paralysis" ComputerHealth,Vol.22,No.10, pp.835.

[6] Xin Liu, Yifan Xia,Hui Yu,Junyu Dong, MuweiJian, Tuan D. Pham,2020" The Current Network ofHierarchyAutomaticparalysisofthefacialnerveAssessment, IEEE Neural Transactions RehabilitationandSystemsVol.28,no.3,p23252332Engineering.

[7] SDN-powered Varun G. Menon, Sunil Jacob, SairaJoseph.EdgeComputingforHumanoidParalyzedAssistance Patients" Things Journal Internet, vol.7,no.7,pp.5874-5957.

[8] Varun Menon, Sunil Jacob, Srija Rajesh,VarghesePaul,P.Vinod,2020"Securebraintobrain"EdgeComputing Communication Paralyzed to help post-stroke The internet of patients" Patients ReportersWithoutBorders, vol.7.

[9] J.More,S.Patil,JitendraM.More,PrayagS.Patil, Satish S. Marathe,2020" COVID 19

ShreerangCentre'scare,RecentInternationalJournalEngineeringandTechnology(IJ RTE),Vol.9,no.3,pp

[10] The Facebook IoT-based smart wheelchair forJenniferDSouza,SanketSrivatsava,RuthPrithika,Sahana.WearableHealthCareS ensorParalyticPatientAssistanceSystemOngoingmonitoringofhealth"RecentIntern ationalJournalEngineeringandTechnology(IJRTE).

[11] KomalB.Sindagi,M.B.Mulik,2020"IOT"RukkayyaL.MujawarPatientObservation SystembasedonIOT,"InternationalEngineeringandTechnology Researcher Journal" (IRJET), Vol.7, No.6,pp. 